\title{
Difficult diagnosis: The Stewart-Treves syndrome vs. Kaposi sarcoma following mastectomy for breast cancer - A case report
}

\author{
Daniel Timofte ${ }^{1}$, Roxana Maria Livadariu* ${ }^{\star 1}$, Radu Danila ${ }^{1}$, Corneliu Diaconu ${ }^{1}$, Delia- \\ Gabriela Ciobanu-Apostol ${ }^{2}$
}

${ }^{1}$ Department of Surgery, III-rd Surgical Unit, "Sf. Spiridon" University Hospital, "Grigore T. Popa" University of Medicine, Iasi, Romania, ${ }^{2}$ Department of Pathology, "Sf. Spiridon" University Hospital, "Grigore T. Popa" University of Medicine, lasi, Romania

\begin{abstract}
The Stewart-Treves syndrome is defined as lymphangiosarcoma of an extremity appearing due to chronic lymphedema, classically following radical mastectomy for breast cancer. We report the case of a 67 years old women admitted for an angiosarcoma of the right forearm occurring 14 years after a modified radical mastectomy and adjuvant chemotherapy for carcinoma of the right breast. A wide excision of the lesion and split-thickness skin graft was performed with uneventful recovery and satisfactory healing of the graft tissue and functional aspect of the arm. The pathological examination showed conventional high-grade angiosarcoma with a mixture of vasoformative features, whereas the immunohistochemical tests showed Ki67 positive expression in $70 \%$ of tumor, CD31 and CD34 intense positive, Factor 8 positive and HHV8 intense diffuse at nuclear level which advocates for nodular Kaposi sarcoma. Further on, the D2-40 and Flt-4 (VEGF-R3) detection were positive on lymphatic endothelium. Lymphangiosarcoma is a rare and aggressive tumor with a very poor prognosis. Early clinical diagnosis and histological confirmation may pose extreme difficulties. Moreover, the clinician should be aware of this rare pathological entity and regard it in the diagnosis algorithm of the patients with nodules linked to chronic lymphedema. It is worth mentioning that in our experience of more than 1100 cases of breast cancer diagnosed, operated and followed by the same surgical team in the last 10 years, this is the first Stewart- Treves syndrome versus Kaposi sarcoma case we encountered.
\end{abstract}

Keywords: Stewart - Treves syndrome, Kaposi sarcoma, lymphangiosarcoma, lymph edema, mastectomy

\section{Introduction}

The Stewart-Treves syndrome is defined as an angiosarcoma of an extremity appearing due to chronic lymphedema. Although classically described as related to chronic lymphedema after radical mastectomy for

Received: May 2015; Accepted after review: June 2015; Published: June 2015.

*Corresponding author: Roxana Maria Livadariu, MD, III-rd Surgical Unit, "Sf. Spiridon" University Hospital, Independentei Boulevard no. 1, code 700111, lasi, Romania.

Email: roxanalivadariu@yahoo.com breast cancer, there have been reported cases of angiosarcoma developed in morbidly obese patients that were thought to be the result of derangements in lymphatic drainage secondary to excessive adipose tissue [1]

There are also reported cases of angiosarcoma of congenital nature and other causes of chronic secondary lymphedema, such as posttraumatic or post radiation lymphedema and also cases of upper limb angiosarcoma after breast-conserving therapy [2-4]. 
Stewart-Treves syndrome consists of lymphangiosarcoma classically described as purplish to brownish blotch and cutaneous nodular lesions progressing to ulceration in a lymphedematous arm within an average of ten years following mastectomy [5].

Lymphangiosarcoma has a poor prognosis. Disease course can be complicated by recurrent episodes of erysipelas and deep venous thrombosis of affected areas. Patients often require hospitalization due to the occurrence of multiple local recurrences and pulmonary metastasis. Given the aggressive nature of the tumor, high rate of local recurrence, and tendency for early and multiple metastases, long-term survivorship is rare with a mean survival length of 24 months and a 5 -year survival rate of $10 \%$ [6].

Although liver and bone metastases may occur, spread of disease to the lung or chest wall is often the cause of death in patients with Stewart-Treves syndrome. Untreated patients usually live for 5 to 8 months after diagnosis [7].

Kaposi sarcoma shares similarities to Stewart-Treves syndrome and both of them may arise on lymphedema. The coexistence of lymph stasis and vascular oncogenesis is not a coincidence. Due to the impaired lymphatic circulation a regional immunodysfunction appears and this is considered the classical mechanism on Kaposi sarcoma [7].

The case was investigated by routine histopathological exam and immunohistochemistry (IHC). Serial section 4 $\mu \mathrm{m}$ thick were cut from paraffin blocks, stained with hematoxylin eosin (HE) and special stains (PAS, Perls, and van Gieson) and prepared for IHC exam (streptavidin- biotin - peroxidase technique) using appropriate positive and negative controls. Primary antibodies, clone and dilution are presented in Table 1. The reaction was developed using Dako En Vision system for detection.

The percentage of Ki67 positive tumor cells was used as the proliferation index. For the other antibodies the immunostaining pattern was scored as positive (strong staining), weakly positive (faint staining) or negative (absence of staining), and the percentage of positive neoplastic cells was counted.

Table 1. Antibodies used for immunohistochemical study

\begin{tabular}{|c|c|c|c|}
\hline Antibody & Clone & Dilution & Staining pattern \\
\hline CD31 & Clone JC70A, DAKO, Denmark & $1: 20$ & $\begin{array}{l}\text { Membranous and } \\
\text { cytoplasmic staining }\end{array}$ \\
\hline CD34 & $\begin{array}{l}\text { Clone Clone QBEnd 10, DAKO, } \\
\text { Denmark }\end{array}$ & $1: 40$ & $\begin{array}{l}\text { Membranous and } \\
\text { cytoplasmic staining }\end{array}$ \\
\hline Factor VIII & $\begin{array}{l}\text { Polyclonal, Clone Clone F8/86, } \\
\text { DAKO, Denmark }\end{array}$ & $1: 200$ & Cytoplasmic staining \\
\hline D2-40 & $\begin{array}{l}\text { Clone D2-40, M3619, DAKO, } \\
\text { Denmark }\end{array}$ & $1: 100$ & $\begin{array}{l}\text { Membranous and } \\
\text { cytoplasmic staining }\end{array}$ \\
\hline $\begin{array}{l}\text { VEGF-R3 } \\
\text { (p-FIt-4) }\end{array}$ & $\begin{array}{l}\text { Polyclonal, SU 5416, Santa Cruz, } \\
\text { USA }\end{array}$ & $1: 300$ & Cytoplasmic staining \\
\hline HHV8 & $\begin{array}{l}\text { Monoclonal HHV8-LNA-CE-S, } \\
\text { Novocastra, Germany }\end{array}$ & $1: 50$ & Nuclear staining \\
\hline Ki67 & $\begin{array}{l}\text { Monoclonal KI67-MM1-L-CE-S, } \\
\text { Novocastra, Germany }\end{array}$ & $1: 100$ & Nuclear staining \\
\hline
\end{tabular}




\section{Case report}

A 67 years old woman was hospitalized in our clinic for a swelling of the right forearm that appeared 9 months ago. She has a history of modified radical mastectomy (Madden operation) 14 years ago for infiltrating ductal carcinoma of the right breast- T2NoMo; followed by 6 cures of chemotherapy and antiestrogen therapy (Tamoxifen) for 5 years; no radiotherapy was commenced.

The patient declares the gradual increase in size of the swelling described and complains of non-radiating, moderate burninglike pain of the area; further on she noticed the appearance of several nodules along the anterior face of the forearm that ulcerated in the last 6 weeks. An ultrasound and CT scan of the forearm performed on outpatient basis 6 months ago revealed chronic cellulitis; therefore she received a topical antiinflammatory treatment.

The local physical examination performed at the time of the hospitalization in our clinic showed the presence of mild lymphedema of entire right arm and purple spots from the elbow to the wrist. On the anterior face of the forearm, a $20 \times 8 \mathrm{~cm}$ purple area with multiple nodular lesions, tending to confluence, some of them ulcerated, with surrounding erythema and induration were noted; apparently, on clinical examination, the subcutaneous tissue of the described area was infiltrated (Figure 1). We did not find any motor or sensitivity, vascular (peripheral arteries) changes from the right shoulder joint to the right fingers.

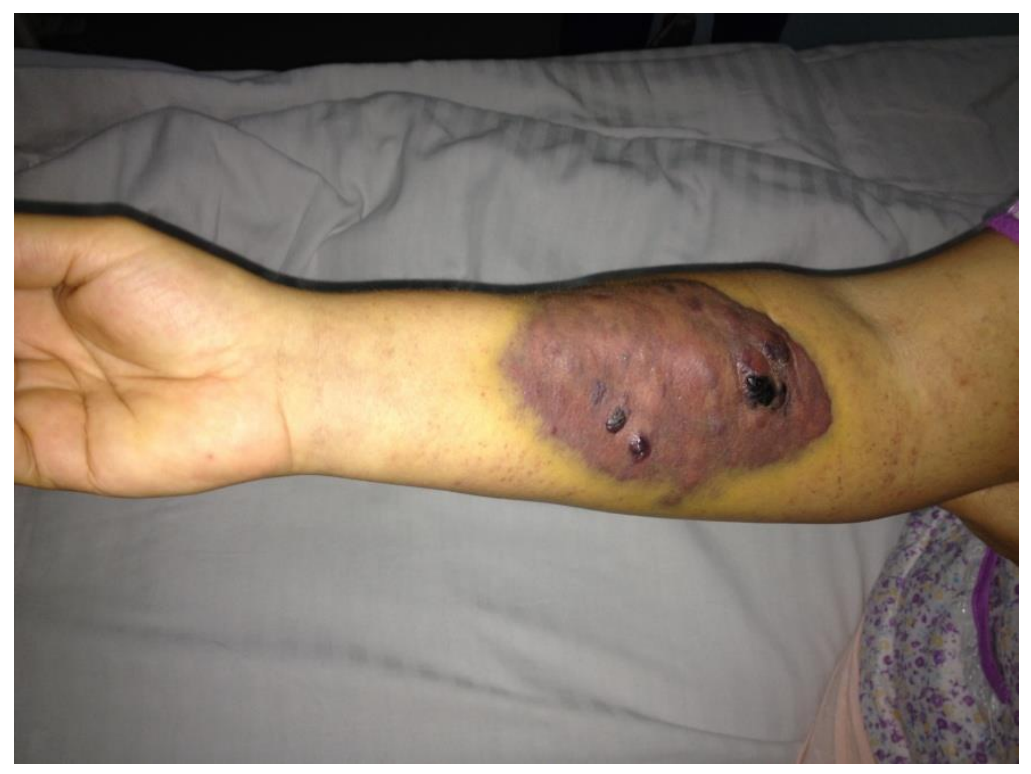

Fig. 1. Preoperative aspect of the tumor: purple area with multiple nodular lesions

The actual blood tests showed no modifications. Abdominal-thoracic CT scan and bone scintigraphy did not reveal any pathological modification related to the current condition or to the breast cancer from her history. PET scan and MRI scan were not available.

Under these circumstances, a wide excision of the lesion and split-thickness skin graft was performed under general anesthesia. The postoperative recovery was uneventful with satisfactory healing of the graft tissue and functional aspect of the arm.

The histopathological examination showed an expansion by a relatively circumscribed, variable cellular proliferation of neoplastic spindled cells arranged in fascicles, a conventional high-grade angiosarcoma, with proliferating vascular channels that dissect dermal collagen and adipose tissue, with prominent tumor necrosis and epidermis ulceration (Figures 2-5). 


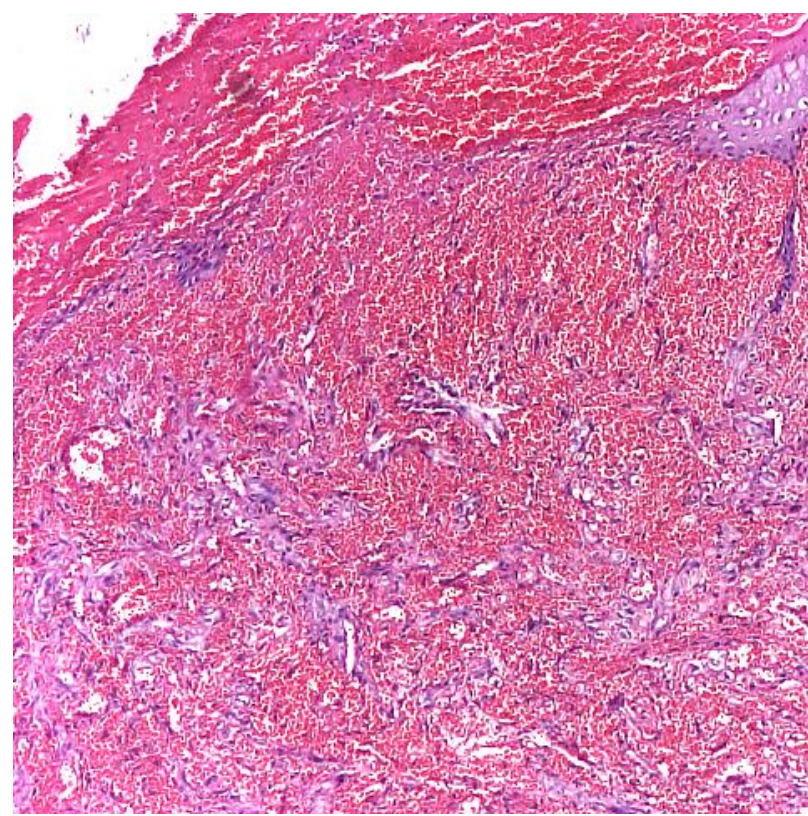

Fig. 2. Epidermal ulceration with diffuse dermal vascular infiltrate accompanied by hemorrhage (HE, $\mathrm{x} 40$ )

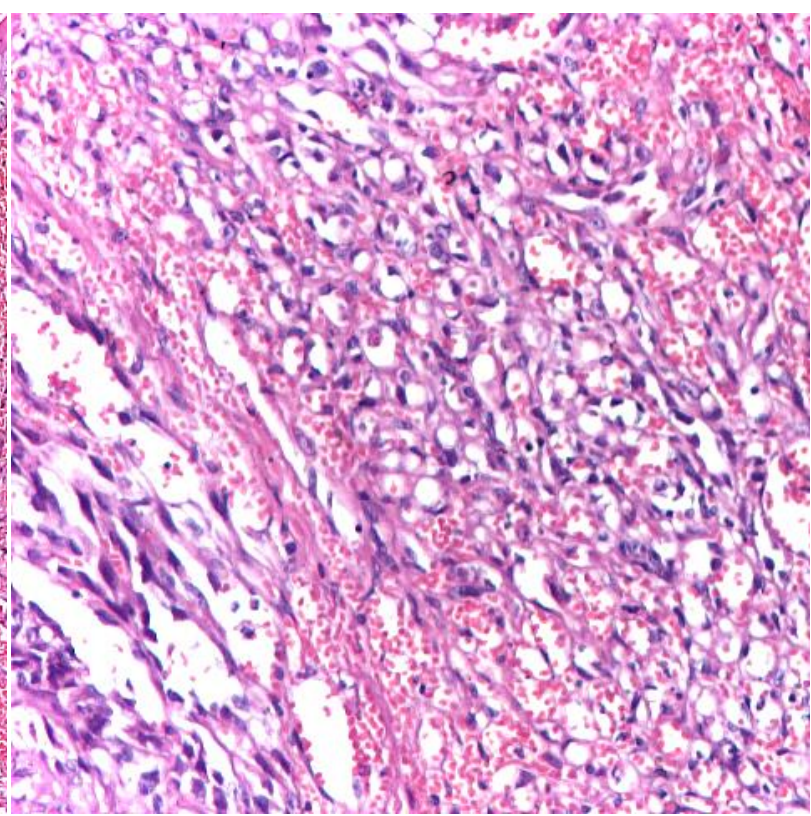

Fig. 3. Fascicles of atypical spindled or epithelioid cells, with slit-like channels containing erythrocytes (HE, x100)

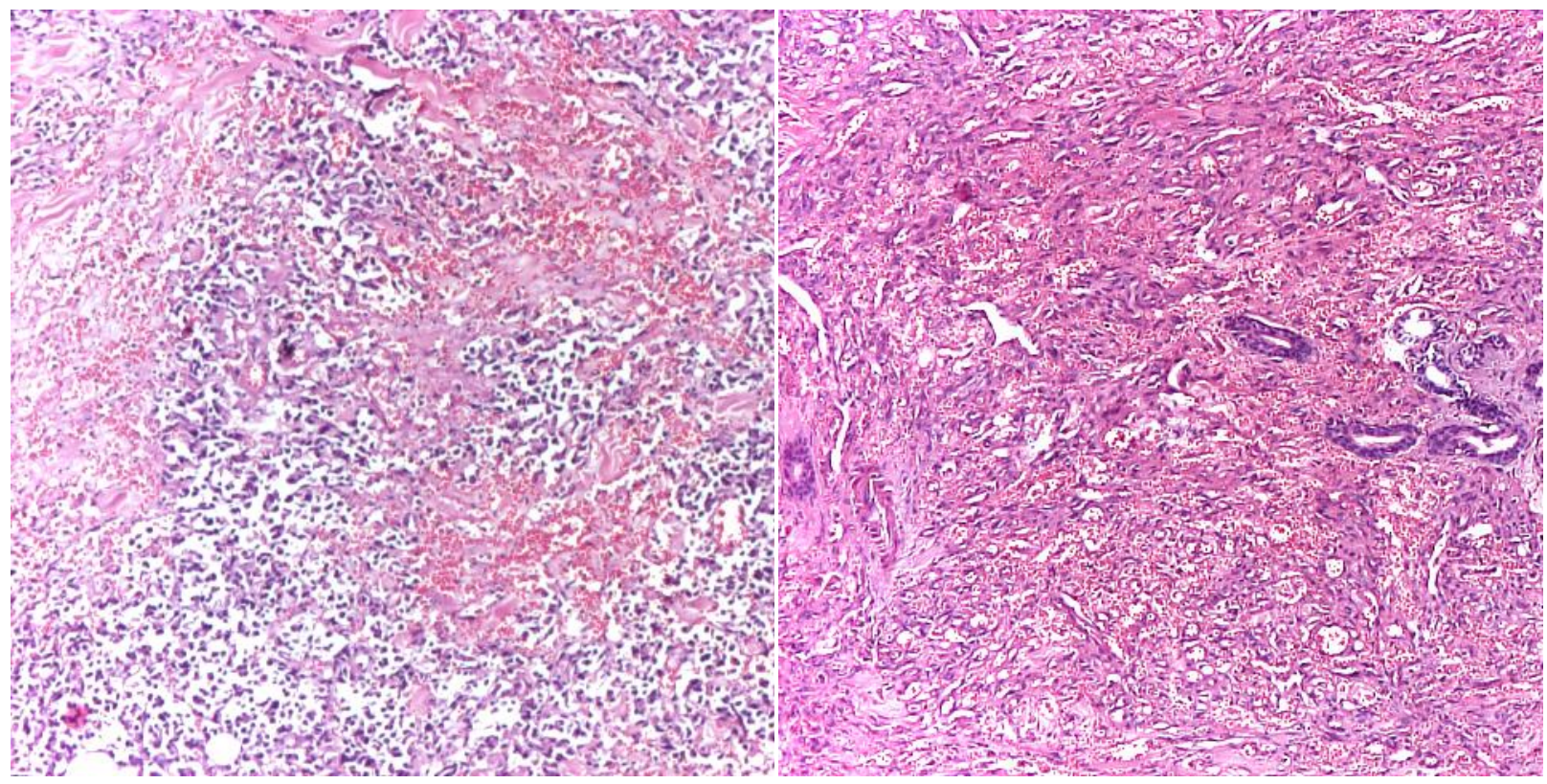

Fig. 4. Tumor necrosis and marked cellular atypical

Fig. 5. Infiltration of skin appendage (HE, x40) $(\mathrm{HE}, \mathrm{x} 40)$

Tumor exhibited a mixture of vasoformative features, including intravascular infiltration (Figure 6). The endothelial cells that lining these channels display hyperchromatism and pleomorphism, have round or oval aspect, sometimes protuberant (Figures 7 and 8).
Erythrocytes are contained within slit-like channels between the individual spindled cells, and in interstitial area with hemosiderin deposits, Perls positive (Figure 9). 


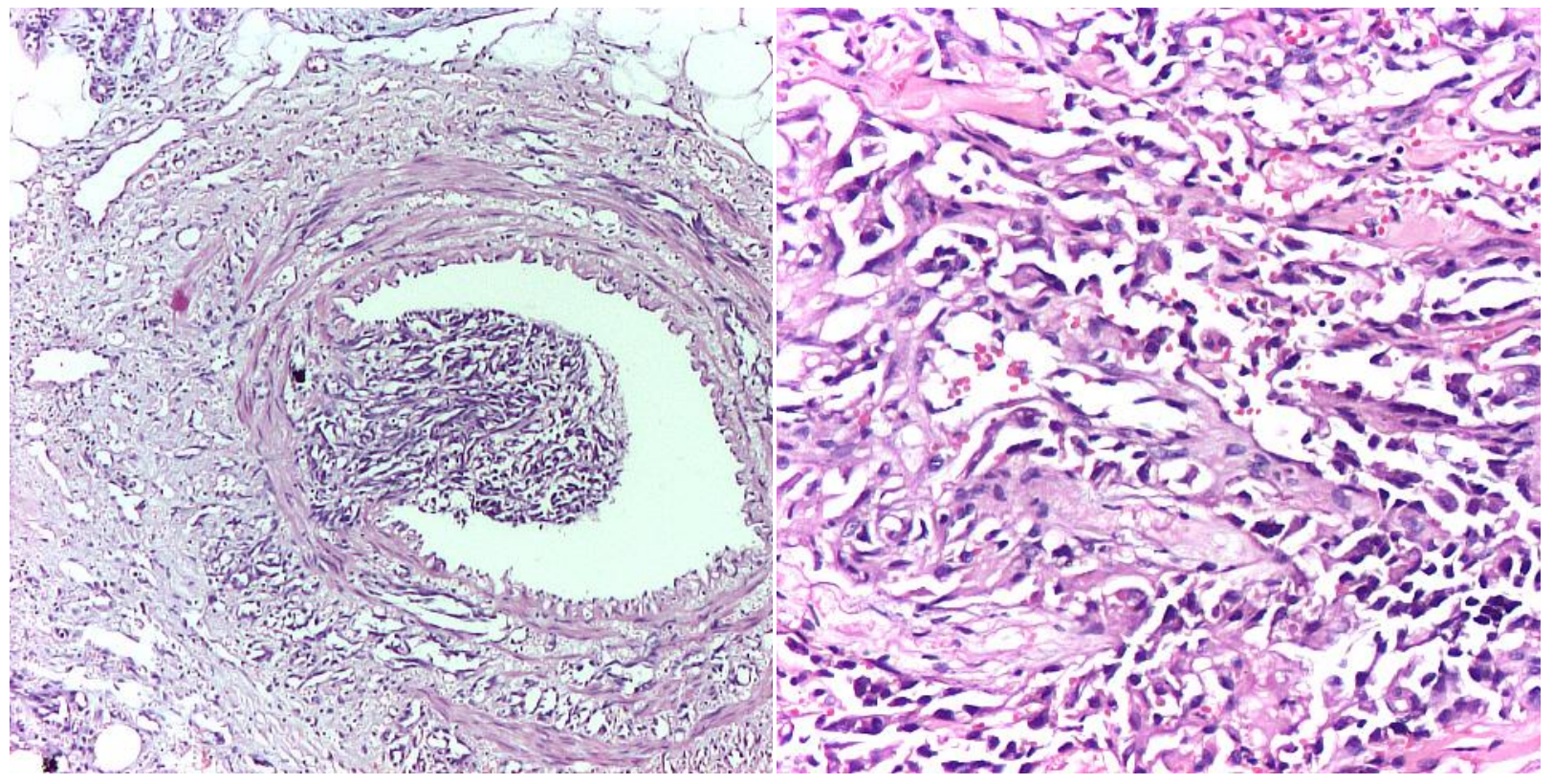

Fig. 6. Newly formed vessels protruding into a larger vascular space (HE, x40)

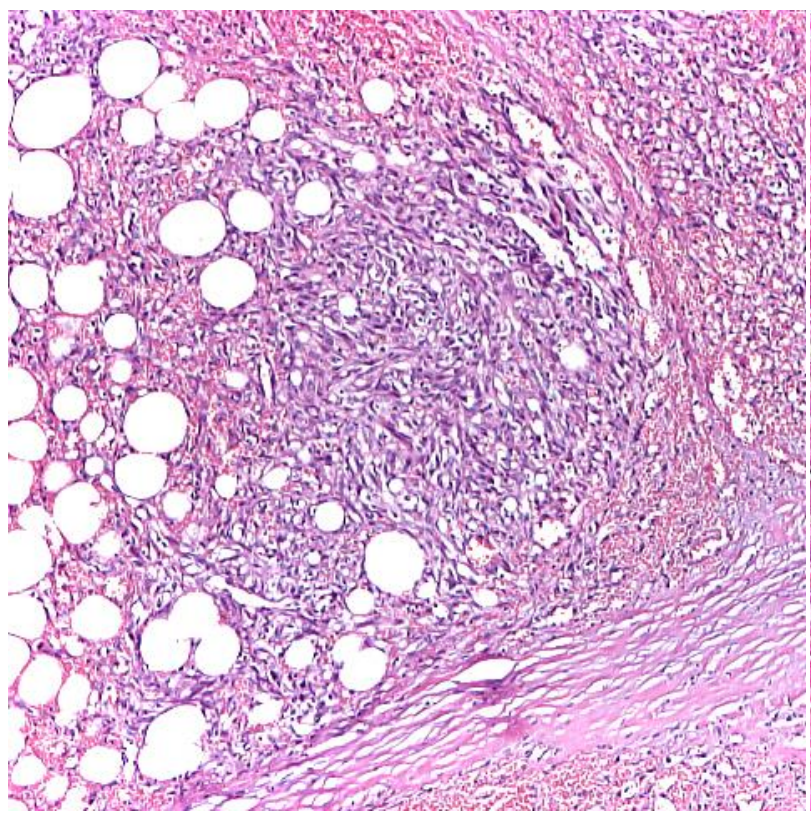

Fig. 8. Solid, haphazard proliferation of atypical spindled cells in hypoderm (HE, x40)

Immunopathological confirmation was, at times, necessary for diagnosis. Antibodies against factor VIII related antigen, CD31 and CD34 were positive in endothelial cells (Figures 10-13), and the mitotic index was very high (70\%) (Figures 14 and 15).
Fig. 7. Papillary projections in vascular lumens (HE, $\mathrm{x} 40$ )

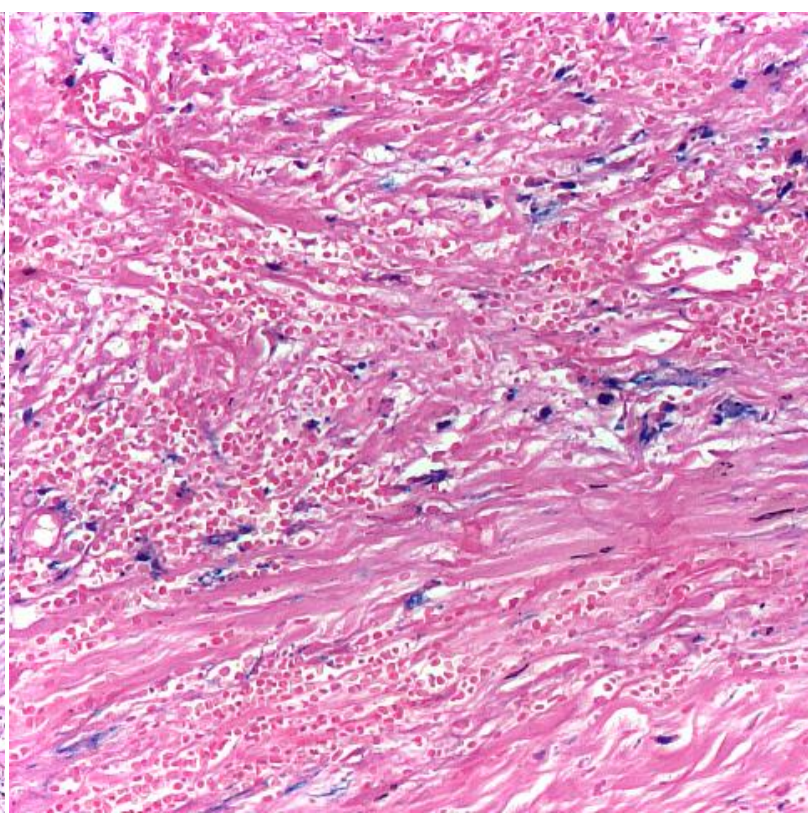

Fig. 9. Hemosiderin pigment with focal distribution (Perls, x100)

HHV8 showed a diffuse, intense nuclear staining and podoplanin D2-40 and Flt-4 a cytoplasmic staining in more than $70 \%$ from tumor vascular population (Figures 16-19). 


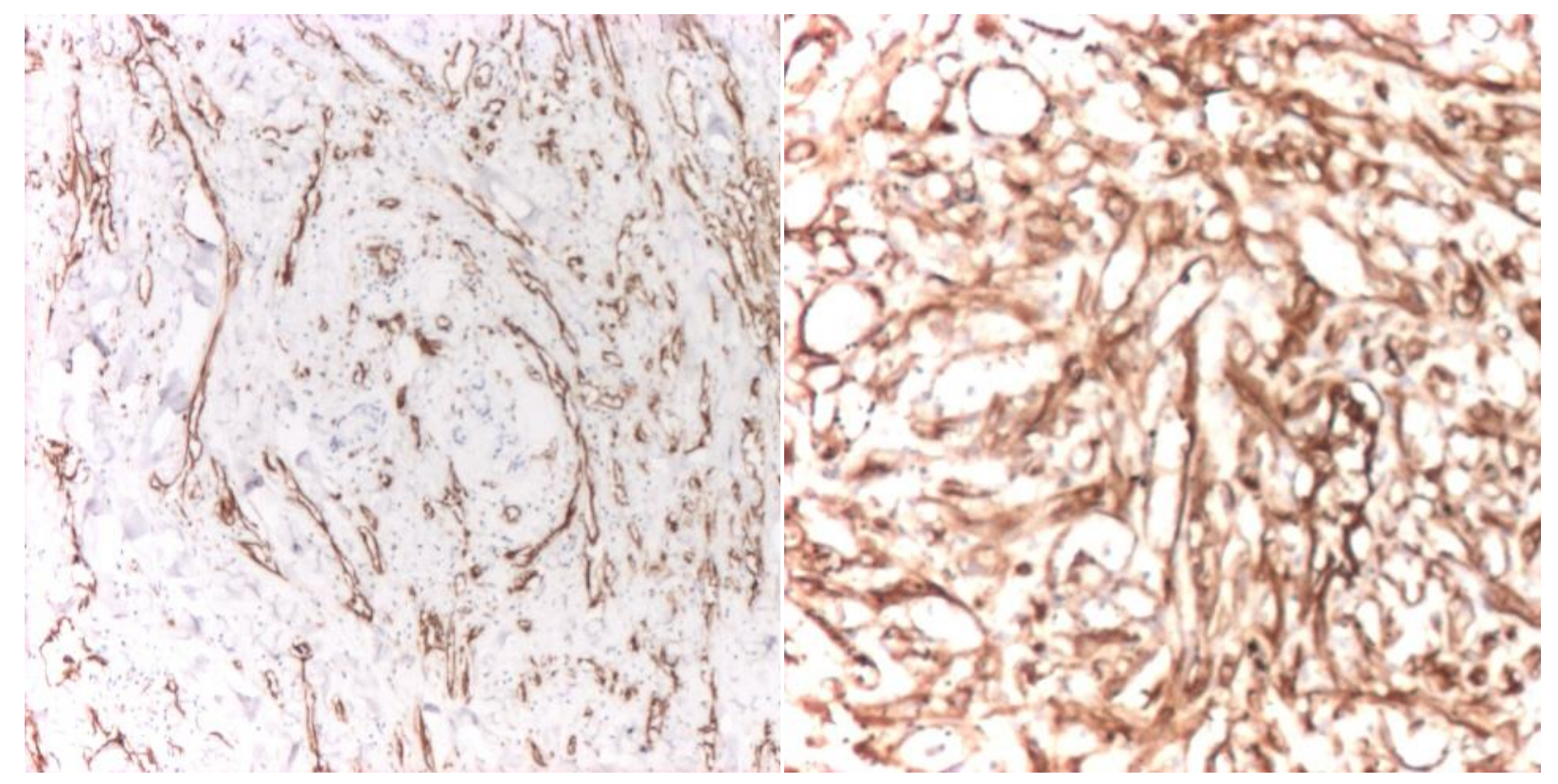

Fig. 10. Irregular vascular spaces CD31 intense positive (IHC, anti-CD31 Ab, x40)

Fig.11. CD34 positive angiosarcoma (IHC, anti-CD34 $\mathrm{Ab}, \mathrm{x100)}$

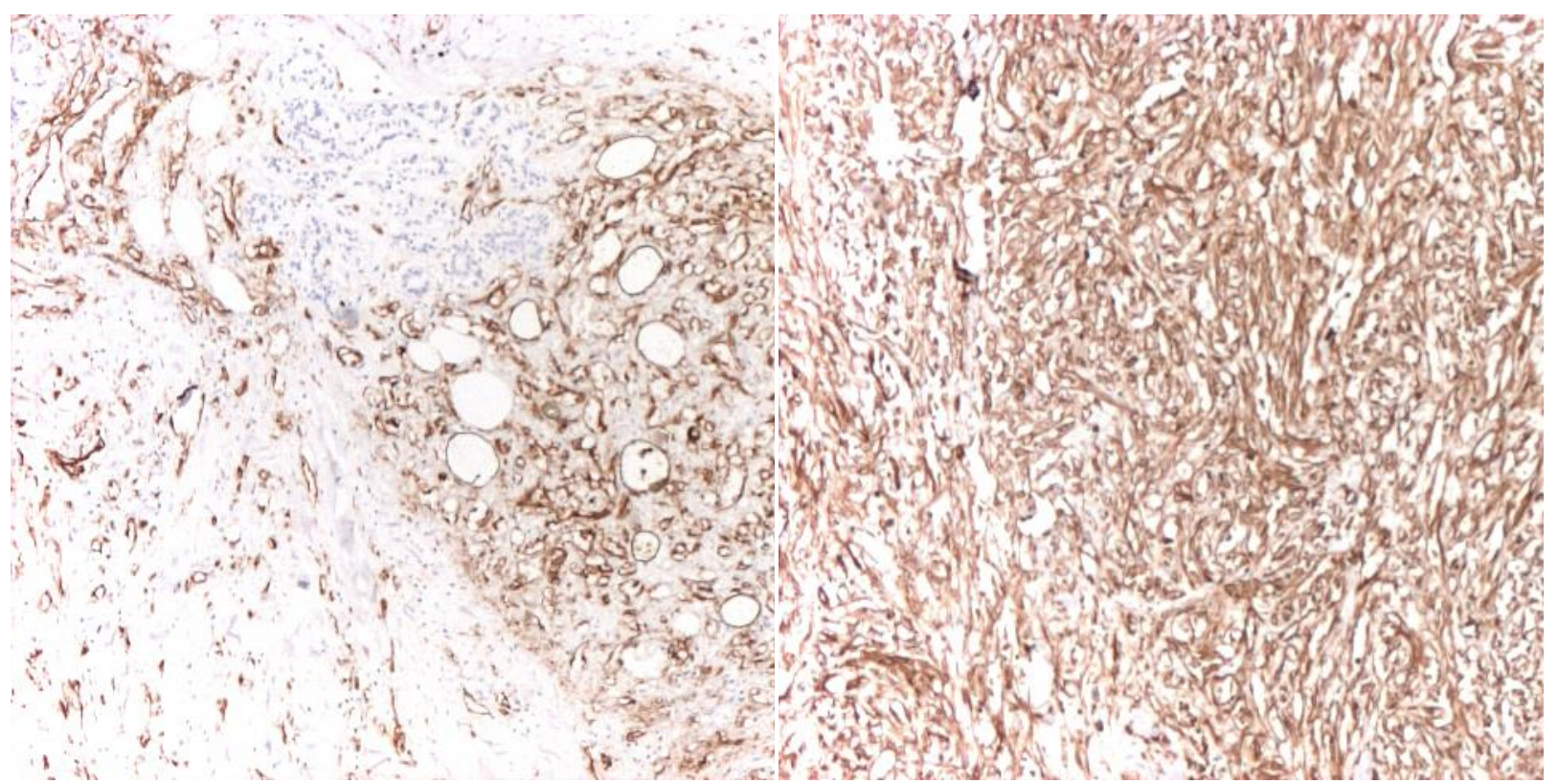

Fig. 12. Infiltration of skin appendage (IHC, anti-CD31 $\mathrm{Ab}, \mathrm{x} 40$ )
Fig. 13. Intense positive staining with CD34 in tumor vessels (IHC, anti-CD34 Ab, x40) 


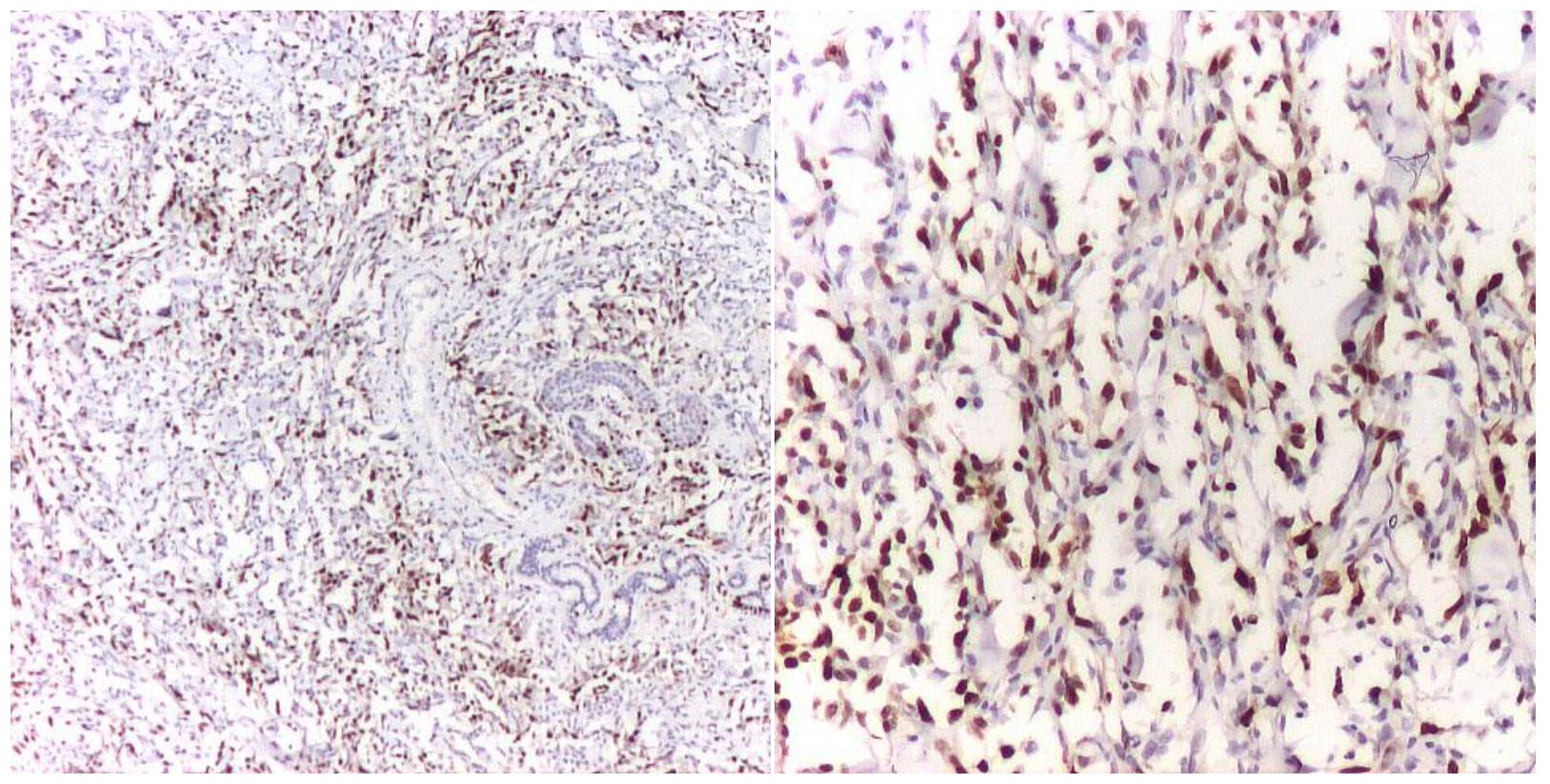

Fig. 14. High proliferative index with $\mathrm{Ki}-67$ (IHC, antiKi67 Ab, x40)

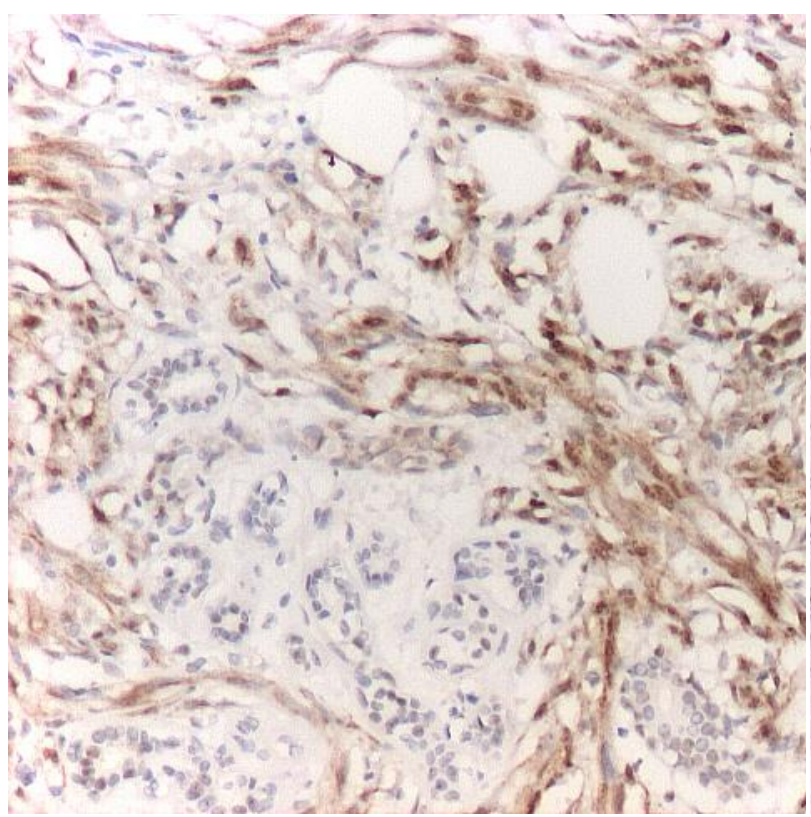

Fig. 15. High proliferative index with Ki-67 (IHC, antiKi67 Ab, x100)

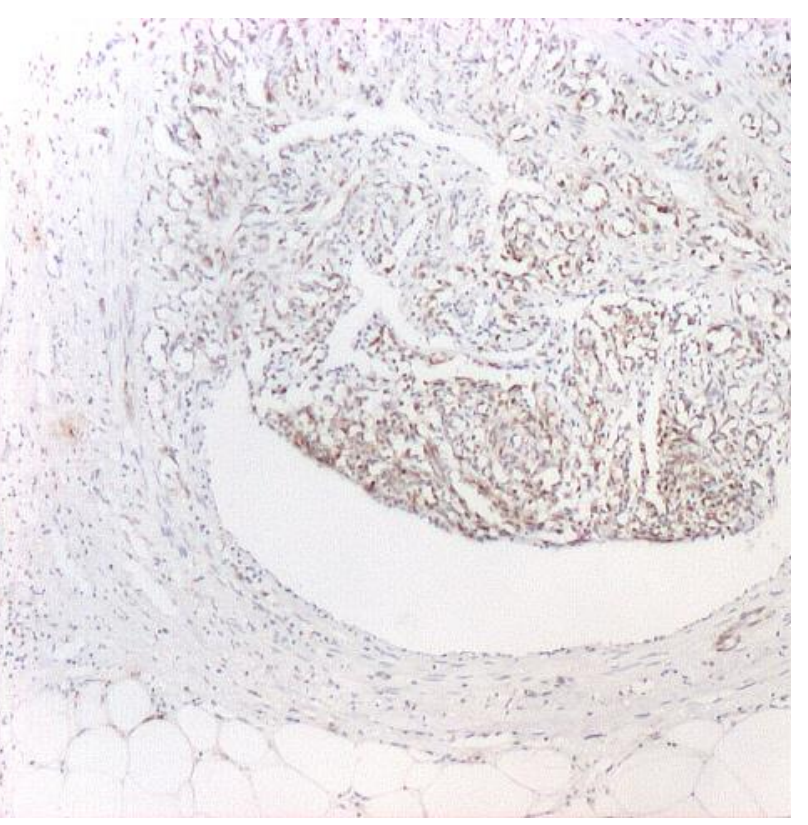

Fig. 16. Positive nuclear staining with HHV - 8 in tumor vessels (IHC, anti-HHV8 Ab, x100)
Fig. 17. Positive staining with HHV - 8 in tumor intravascular proliferation (IHC, anti-HHV8 Ab, x40) 


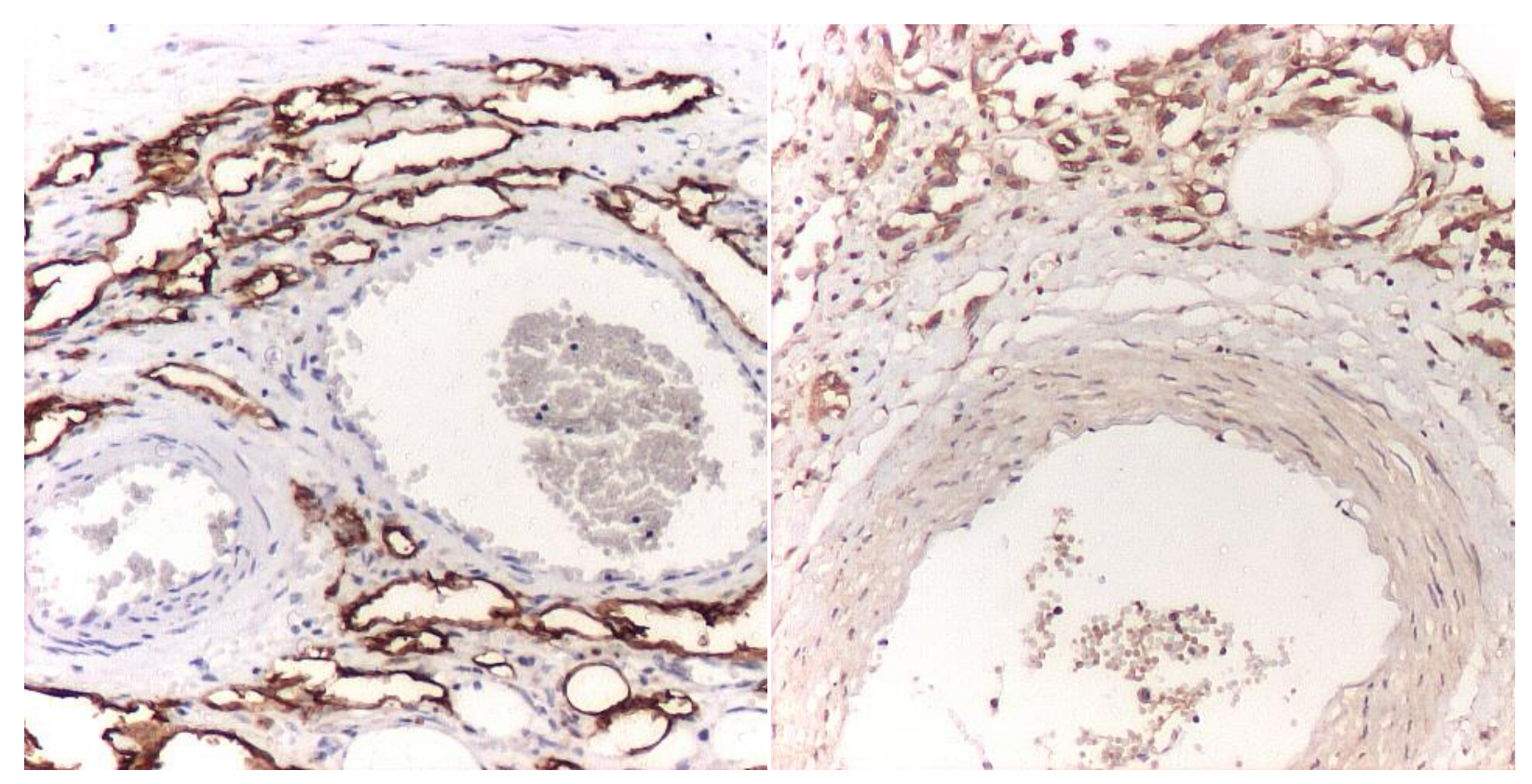

Fig 18. Positive staining with D2 - 40 in tumor vessels with negative staining in mature vessels (IHC, anti-D2$40 \mathrm{Ab}, \mathrm{x} 100)$

Considering the pathology report, the oncology board recommended adjuvant chemotherapy with docetaxel $75 \mathrm{mg} / \mathrm{m} 2$.

\section{Discussions}

Angiosarcoma is a rare and aggressive tumor with a very poor prognosis, with a low disease-free survival at 5 years (35\%) [8]. Early clinical diagnosis and histological confirmation may pose extreme challenges. Moreover, the clinician should be aware of this rare pathological entity and regard it in the diagnosis algorithm of the patients with nodules linked to chronic lymphedema. The Stewart-Treves syndrome has been firstly associated with long-term lymphedema of the superior limb following mastectomy for neoplasms or even congenital [7, 9-12]. Supplementary data has also proved that homograft skin survived much longer if it was transplanted to a lymph edematous limb than to a healthy arm [7]. Even if several reports confirm that the first description of the StewartTreves syndrome as lymphangiosarcoma is correct, the pathological mechanism by which
Fig. 19. Positive staining with Flt-4 in tumor vessels with negative staining in mature vessels (IHC, anti-Flt$4 \mathrm{Ab}, \mathrm{x} 40$ )

lymphedema could induce angiosarcoma is yet a matter of debate [7]. The idea of an existing systemic carcinogenic factor combined with local immunodeficiency was also considered [7].

Kaposi sarcoma shares some similarities to Stewart-Treves syndrome the former being associated with chronic lymphedema but not so aggressive [7, 13]. For a long time, the precise origin of the Kaposi sarcoma has raised divergent points of view sustaining the involvement from different type of cells (endothelial, smooth muscle, undifferentiated mesenchymal cells) [14]. Nowadays, the endothelial differentiation is recognized, although controversies regarding the lymphatic or vascular phenotype still persist [14]. Moreover, the contribution of the local immunodeficiency in the pathogenicity of the Kaposi sarcoma is sustained by evidences of the presence of HHV8 factor, that sustain a direct relationship between the immune response and lymphatic drainage $[15,16]$. Specific markers for lymphatic vessels are expressed in both lymphangiosarcoma and Kaposi sarcoma, therefore a differential 
diagnosis between the two entities is absolutely necessary $[7,17]$. It is worth mentioning that $\mathrm{IHC}$ and supplementary molecular tests prove the presence of HHV8 in almost all cases of Kaposi sarcoma, this marker being absent in lymphangiosarcoma [7].

The histopathological examination algorithm follows successive steps. Firstly, we performed IHC by using anti-CD31, -CD34 and -HHV8 antibodies, the positive results suggesting an angiosarcomatous proliferation, Kaposi-like. Due to the discrepancy between these results and the clinical data indicating a Stewart-Treves syndrome (based on the radical mastectomy, axillar resection and lymphedema developed during several years), the investigation was complemented with antiD2-40 and anti-VEGFR-3 (FIt-4) antibodies, in order to differentiate the vascular origin from the lymphatic one. These markers were also positive.

The current case expresses an intricate immunostaining both for Kaposi sarcoma and lymphangiosarcoma. These findings should be interpreted according to the mainstream publications that highlight the difficulty of the pathological diagnosis of vascular malignancies, even if they are characterized using IHC. The triple positivity for CD31, CD34 and HHV8 can sustain the Kaposi sarcoma diagnosis.

Several histological types are described for the Kaposi sarcoma, in correlation with the clinical aspects (patch, plaque, and nodule). The histological type associated with limb lymph edema is known as lymphangioma-like Kaposi sarcoma [18]. The main morphological features are bulous lesions and marked acanthosis with reactive hyperkeratosis in the epidermis, and large, irregular, anastomosed vascular channels, separated through collagen bundles in the dermis. We could not identify the changes in epidermis, due to the extended skin ulceration, but the tumor proliferation was almost similar to the above description, the small vascular channels being associated with abundant hemorrhage and hemosiderin pigment. According to the literature, this form can be also positive for the lymphatic markers, as D2-40 [18]. Consequently, we consider the use of Flt-4, a more specific and sensitive marker for lymphatic vessels, as compulsory.
The positive reaction for Flt-4 in over $70 \%$ of tumor cells proved the lymphatic differentiation of the small tumor vessels. The publications mainstream includes similar diagnostic difficulties in a series of 16 cases [19], with concomitant HHV8 and D2-40 positivity of $100 \%$, and in two cases with Kaposi sarcoma mimicking Stewart-Treves syndrome, with HHV8 positivity [20]. Based on the general immunohistochemical profile, the final diagnosis was Kaposi sarcoma with endothelial lymphatic differentiation. Therefore, we consider that the results registered in the present case can be interpreted as supplementary proofs confirming the possibility of tumor development starting from the undifferentiated, pluripotent cells, with double differentiation capacity, vascular and lymphatic.

A brief review of the literature reveals controversial data regarding the value of the markers used in the diagnosis of the tumours with vascular or lymphatic origin. Therefore, we consider the following examples as relevant. The HHV8 specificity and sensibility is increased by its positivity in Kaposi-like lesions present in sarcoma, and decreased by its negativity in angiosarcoma, kaposiform hemangioendothelioma, hemangioma with fusiform cells, reactive angioendothelioma or reactive vascular proliferations $[14,21]$. Reffering to VEGF-R3 (as a member of the VEGF family), its expression is restricted to the lymphatic endothelium and, consequently, is related to the lymphangiogenesis process [14]. Despite some opinions that sustain the value of vWF, CD34, and CD31 [14, 22-26], VEGFR-3 - due to its specificity for the lymphatic endothelium - can be considered an accurate marker for the confirmation of the lymphatic differentiation in Kaposi sarcoma and cutaneous lymphangioma [14, 24].

From the clinical point of view, as far as surgical treatment is concerned, reported data show that there is no significant difference in survival when comparing patients initially treated with wide excision with patients treated with amputation [27]. Surgical treatment can be preceded or followed by oncological therapy. Locally advanced tumors or metastatic forms can be treated with mono- or polychemotherapy, on systemic or local administration. Cases with good response 
using courses of intra-arterial mitoxantrone (MX) and paclitaxel (PTX) are reported [28, 29].

It is worth mentioning that in our experience of more than 1100 cases of breast cancer diagnosed, operated and followed by the same surgical team in the last 10 years, this is the first case of Stewart-Treves syndrome versus Kaposi sarcoma we encountered. We consider that our preventing post mastectomy upper limb lymph edema management consisting in manual lymph drainage, compression garments, physical therapy and postoperative prescription of Coumarin, decreases the risk of StewartTreves syndrome by decreasing the incidence of post mastectomy lymph edema.

\section{References}

1. Shon $\mathrm{W}$, Ida $\mathrm{CM}$, Boland-Froemming JM, Rose PS, Folpe A. Cutaneous angiosarcoma arising in massive localized lymphedema of the morbidly obese: a report of five cases and review of the literature. J Cutan Pathol 2011; 38(7):560-564.

2. Laguerre $B$, Lefeuvre $C$, Kerbrat $P$, Hassel $M$. Stewart-Treves syndrome arising in posttraumatic lymphedema. Bull Cancer 1999; 86(3):279-282.

3. Fodor J, Orosz Z, Szabo E, et al. Angiosarcoma after conservation treatment for breast carcinoma: our experience and a review of the literature. J Am Acad Dermatol 2006; 54:499-504.

4. Vojtísek R, Kinkor Z, Fínek J. Secondary angiosarcomas after conservation treatment for breast cancers. Klin Onkol 2011; 24(5):382-388.

5. Lee SB, Cho BK, Houh W, Song YT, Shim SI, Choi IB. A case of Stewart-Treves syndrome. J Korean Med Sci 1988; 3(2):83-88.

6. Caldwell JB, Ryan MT, Benson PM, James WD. Cutaneous angiosarcoma arising in the radiation site of a congenital hemangioma. $J$ Am Acad Dermatol 1995; 33(5 Pt 2):865-870.

7. Sharma A, Schwartz RA. Stewart-Treves syndrome: pathogenesis and management. $J$ Am Acad Dermatol 2012; 67(6):1342-1348.

8. Strobbe LJ, Peterse $\mathrm{HL}$, van Tinteren $\mathrm{H}$, Wijnmaalen A, Rutgers EJ. Angiosarcoma of the breast after conservation therapy for invasive cancer, the incidence and outcome.

\section{Conclusions}

The double immunostaining for vascular and lymphatic vessels concomitantly present with the typical immunostaning for Kaposi sarcoma can indicate a rare form of Kaposi sarcoma that occurs after a radical mastectomy and, possible, originates in the multipotent stem cells. The difficulty of diagnosis is provocative, few similar cases being diagnosed as postmastectomy Kaposi syndrome or StewartTreves syndrome with HHV8 positivity. The identification of other markers for endothelial differentiation, with higher sensitivity and specificity, is required for a more accurate diagnosis.

An unforeseen sequela. Breast Cancer Res Treat 1998; 47(2):101-109.

9. Dürr HR, Pellengahr $C$, Nerlich A, Baur A, Maier $M$, Jansson V. Stewart-Treves syndrome as a rare complication of a hereditary lymphedema. Vasa 2004; 33(1):42-45.

10. Hallel-Halevy D, Yerushalmi J, Grunwald MH, Avinoach I, Halevy S. Stewart-Treves syndrome in a patient with elephantiasis. J Am Acad Dermatol 1999; 41(2):349-350.

11. Hulme SA, Bialostocki A, Hardy SL, Tills MR. Stewart-Treves syndrome in a congenitally lymphedematous upper limb. Plast Reconstr Surg 2007; 119(3):1140-1141.

12. Komorowski AL, Wysocki WM, Mitus J. Angiosarcoma in a chronically lymphedematous leg: An unusual presentation of Stewart-Treves syndrome. South Med J 2003; 96(8):807-808.

13. Ron IG, Amir G, Marmur S, Chaitchik S, Inbar MJ. Kaposi's Sarcoma on a lymphedematous arm after mastectomy. Am J Clin Oncol 1996; 19(1):87-90.

14. Folpe AL, Veikkola $T$, Valtola R, Weiss SW. Vascular endothelial growth factor receptor-3 (VEGFR-3): a marker of vascular tumors with presumed lymphatic differentiation, including Kaposi's sarcoma, kaposiform and Dabska-type hemangioendotheliomas, and a subset of angiosarcomas. Mod Pathol 2000; 13(2):180-185.

15. Ruocco E, Ruocco V, Tornesello ML, Gambardella A, Wolf R, Buonaguro FM. Kaposi's sarcoma: etiology and pathogenesis, inducing factors, causal associations, and 
treatments: facts and controversies. Controversies in Dermatology: Part IV 2013; 31(4):413-422.

16. Mallon E, Powell S, Mortimer P, Ryan TJ. Evidence for altered cell-mediated immunity in postmastectomy lymphoedema. Br J Dermatol 1997; 137:928-933.

17. Mankey CC, McHugh JB, Thomas DG, Lucas DR. Can lymphangiosarcoma be resurrected? A clinicopathological and immunehistochemical study of lymphatic differentiation in 49 angiosarcomas. Histopathology 2010; 56(3):364-371.

18. Grayson W, Pantanowitz L. Histological variants of cutaneous Kaposi sarcoma. Diagn Pathol 2008, 3:31-42.

19. Lee.KB, Lee $H S$, Lee $H E$, et al. Immunohistochemical characteristics of Kaposi sarcoma and its mimicries. Korean $J$ Pathol 2006; 40(5):361-367.

20. Allan AE, Shoji T, Li N, Burlage A, Davis B, Bhawan J. Two cases of Kaposi's sarcoma mimicking Stewart-Treves syndrome found to be human herpesvirus-8 positive. $A m \mathrm{~J}$ Dermatopathol 2001; 23(5):431-436.

21. Cheuk W, Wong KOY, Wong CSC, Dinkel JE, Ben-Dor D, Chan JKC. Immunostaining for human herpesvirus 8 latent nuclear antigen-1 helps distinguish Kaposi sarcoma from its mimickers. Am J Clin Pathol 2004; 121(3):335342.

22. Rutgers JL, Wieczorek R, Bonetti F, et al. The expression of endothelial cell surface antigens by AIDS-associated Kaposi's sarcoma: evidence for a vascular endothelial cell origin. Am J Pathol 1986; 122(3):493-499.

23. Facchetti F, Lucini L, Gavazzoni R, Callea F. Immunomorphological analysis of the role of blood vessel endothelium in the morphogenesis of cutaneous Kaposi's sarcoma: a study of 57 cases. Histopathology 1988; 12(6): 581-593.

24. Jussila $L$, Valtola $R$, Partanen $T A$, et al. Lymphatic endothelium and Kaposi's sarcoma spindle cells detected by antibodies against the vascular endothelial growth factor receptor3.Cancer Res 1998; 58(8):1599-1604.

25. Lymboussaki A, Partanen TA, Olofsson B, et al. Expression of the vascular endothelial growth factor C receptor VEGFR-3 in lymphatic endothelium of the skin and in vascular tumors. Am J Pathol 1998; 153(2): 395-403.

26. Sauter B, Foedinger D, Sterniczky B, Wolff K, Rappersberger K. Immunoelectron microscopic characterization of human dermal lymphatic microvascular endothelial cells: differential expression of CD31, CD34, and type IV collagen with lymphatic endothelial cells vs blood capillary endothelial cells in normal human skin, lymphangioma, and hemangioma in situ. J Histochem Cytochem 1998; 46(2):165-176.

27. Grobmyer SR, Daly JM, Glotzbach RE, Grobmyer AJ $3^{\text {rd }}$. Role of surgery in the management of postmastectomy extremity angiosarcoma (Stewart-Treves syndrome). J Surg Oncol 2000; 73(3):182-188.

28. Breidenbach $M$, Rein $D$, Schmidt $T$, et al. Intra-arterial mitoxantrone and paclitaxel in a patient with Stewart-Treves syndrome: selection of chemotherapy by an ex vivo ATPbased chemosensitivity assay. Anticancer drugs 2000; 11(4):269-273.

29. Fujisawa $\mathrm{Y}$, Ito $\mathrm{M}$, Mori $\mathrm{K}$, et al. Intra-arterial mitoxantrone/paclitaxel in angiosarcoma of the lower limb associated with chronic lymphedema (Stewart-Treves syndrome) in a patient with cervical cancer. Eur $J$ Dermatol 2011; 21(1):119-120. 